\title{
Target localization based on joint measurement of amplitude and frequency in a LOFAR field*
}

\author{
Lin-wei TAO, Ying-min WANG \\ (School of Marine Science and Technology, Northwestern Polytechnical University, Xi'an 710072, China) \\ E-mail: tlwlyn@nwpu.edu.cn; ywang@nwpu.edu.cn \\ Received Aug. 7, 2013; Revision accepted Nov. 20, 2013; Crosschecked Jan. 14, 2014
}

\begin{abstract}
To estimate the motion parameters of a moving target before its passing by the closest point of approach (CPA) point in a low frequency analyzing and recording (LOFAR) field, an error-free theoretical method based on the joint measurement of target radiated noise's amplitude and frequency was presented. First, the error-free theoretical equations for target characteristic frequency, absolute velocity, the CPA, and amplitude of the radiation noise were derived by three equal interval measured values of the target amplitude and frequency. Then, the method to improve the calculation accuracy was given. Finally, the simulation and experiments were conducted in the air and showed the correctness of this method. By using one single piece of LOFAR, this method can calculate four target parameters and the relative error of each estimated parameter is less than $10 \%$.
\end{abstract}

Key words: Low frequency analyzing and recording (LOFAR), Closest point of approach (CPA), Doppler frequency shift, Transmission loss

doi: 10.1631 jzus.A1300263

Document code: A

CLC number: TB52

\section{Introduction}

Low frequency analyzing and recording (LOFAR) is a very important application in the passive measurement field. Generally, LOFAR with no directivity can only passively receive radiated noise of the target. Line spectrum information contained in the noise is recorded and processed to calculate the motion parameters of the target. With characteristics of low cost, small volume, light weight, etc., the LOFAR is applied widely in various fields, for example, underwater target localization (Wu and Sun, 1999; Ling and Wang, 2007).

Methods with single pieces of LOFAR for target parameter estimation are as follows. The classical algorithm for LOFAR, namely algorithm of Dopplerclosest point of approach (CPA) was proposed in (Tao 2009). Tao (2009) calculated the velocity of the target

\footnotetext{
* Project supported by the National Natural Science Foundation of China (No. 51209173)

(C) Zhejiang University and Springer-Verlag Berlin Heidelberg 2014
}

and CPA approximately by recording the two symmetrical target frequencies of the CPA point. An improved algorithm of the Doppler-CPA was also proposed in (Tao, 2009), where the calculation of the absolute velocity of the target and the CPA error-free theoretical formula were deduced by introducing time information. All the methods above can only be applied after the target passes by the CPA point, which is limited in use. The approximate calculation method of the target speed and CPA was presented in (Yu, 2011), which used any of the three point of frequency measurements and information of the change rate of the target line spectrum. Actually, the Doppler variation of the target is originally a slow process with noise. Therefore, using the rate of change to calculate the target motion parameters may cause some large errors.

Target localization with joint multiple LOFAR is another active direction, such as the classical LOFAR FIX (LOFIX) principle and analysis of localization accuracy in (Hu et al., 2009), the classical hyperbolic fixing (HYFIX) principle and analysis of localization accuracy in (Sun et al., 2010). The passive range estimation was completed by using a vector sensor array 
(Li et al., 2012). The passive parameter estimation and its accuracy were analyzed from different aspects (Shang et al., 1985; Mao, 2001; Brian and Kam, 2002; Xue et al., 2005; Ma, 2007; Cockrell and Schmidt, 2010). To improve the accuracy of the target frequency measurement, some methods of image processing were applied in previous studies (Fan et al., 2002; Yang et al., 2010).

This paper focuses on using a single piece of LOFAR. The radiated noise's amplitude of the target at any time (not restricted to passing by the CPA necessarily) is collected by three types of equal intervals. The error-free calculation formulas for the four types of status information consisted of absolute velocity of the target, characteristic frequency of the target, the closest distance, and the absolute amplitude, are then derived.

\section{Measurement model}

\subsection{Measurements of frequency}

As shown in Fig. 1, the target moves with speed $v$, and the CPA is $D$. The characteristic frequency of the line-spectrum is $f_{\mathrm{T}}, t_{i}$ is measure time, $r_{i}$ is distance from LOFAR to target, and $\varphi_{i}$ is the relative bearing between the moving forward direction of the target and LOFAR in the $i$ th measurement, $i=1,2,3$.

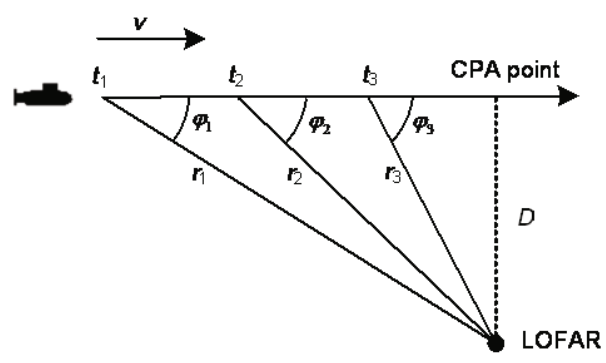

Fig. 1 Motion diagram between target and LOFAR

By the Doppler shift equation, the line-spectrum frequency of the detected target by using LOFAR is (Tao and Wang, 2008)

$$
f_{i}=f_{\mathrm{T}}+f_{\mathrm{T}} \frac{v}{c} \cos \varphi_{i}
$$

where $f_{i}$ is the $i$ th measured target frequency, and $c$ is the speed of sound.

\subsection{Measurements of amplitude}

LOFAR is mainly used for recording and processing the strong line spectrum in the noise radiated by the target. According to (Liu and Lei, 1993), the line spectrum noise mainly consists of mechanical noise and propeller noise in the background noise of the target. The frequency ranges from $10 \mathrm{~Hz}$ to $2000 \mathrm{~Hz}$.

Regarding the target as a point source, according to the spherical wave theory of sound propagation, the transmission loss is

$$
\mathrm{TL}=20 \lg r+\alpha r
$$

where $\alpha$ is the absorption coefficient. The first item of the transmission loss is extended loss, and the second item is sound absorption loss.

The empirical formula of the low frequency band absorption coefficient which is derived from Liu and Lei (1993) is

$$
\alpha=\frac{0.102 f^{2}}{1+f^{2}}+\frac{40.7 f^{2}}{4100+f^{2}} .
$$

The absorption coefficient is $0.000279-0.12$ in the frequency band of 10-2000 Hz. If the distance is $10 \mathrm{~km}$, the extended loss will be $80 \mathrm{~dB}$, and the maximum absorption loss is $1.2 \mathrm{~dB}$. Therefore, the absorption loss can be ignored when the frequency is in the low band.

Assuming that there are only extended loss and expansion of spherical waves, the amplitude data of the target noise which is received by LOFAR are (Liu and Lei, 1993)

$$
|p|=\frac{A}{r}
$$

where $p$ is the pressure of sound, and $A$ is the amplitude of sound pressure which is equivalent to the unit distance from the target.

\subsection{Equation of observation}

As shown in Fig. 1, the frequency and amplitude of the target are measured and recorded at equal interval times $t_{1}, t_{2}$, and $t_{3}\left(t_{2}-t_{1}=t_{3}-t_{2}=\Delta t\right)$. The measurement of frequency is shown in Eq. (1). 
The measurement of amplitude is

$$
p_{i}=\frac{A_{\mathrm{T}}}{r_{i}}=\frac{A_{\mathrm{T}} \sin \varphi_{i}}{D},
$$

where $p_{i}$ is the signal amplitude of the target in the $i$ th measurement, $i=1,2,3 . A_{\mathrm{T}}$ is the absolute amplitude which is equivalent to the unit distance from the target. According to the geometric principle,

$$
v \Delta t=\frac{D}{\operatorname{tg} \varphi_{1}}-\frac{D}{\operatorname{tg} \varphi_{2}}=\frac{D}{\operatorname{tg} \varphi_{2}}-\frac{D}{\operatorname{tg} \varphi_{3}} .
$$

\section{Calculation of the target's parameters}

\subsection{Characteristic frequency}

Eq. (1) can be rewritten as

$$
\cos \varphi_{i}=\frac{f_{i}-f_{\mathrm{T}}}{f_{\mathrm{T}}} \frac{c}{v},
$$

and Eq. (5) can be rewritten as

$$
\sin \varphi_{i}=\frac{p_{i} D}{A_{\mathrm{T}}},
$$

then

$$
\operatorname{tg} \varphi_{i}=\frac{p_{i} D}{A_{\mathrm{T}}} \frac{f_{\mathrm{T}}}{f_{i}-f_{\mathrm{T}}} \frac{v}{c} .
$$

Substituting Eq. (9) into Eq. (6), and substituting the measured values of $t_{1}, t_{2}$, and $t_{3}$, we have

$$
\frac{f_{1}-f_{\mathrm{T}}}{p_{1}}-\frac{f_{2}-f_{\mathrm{T}}}{p_{2}}=\frac{f_{2}-f_{\mathrm{T}}}{p_{2}}-\frac{f_{3}-f_{\mathrm{T}}}{p_{3}} .
$$

Because $f_{1}, f_{2}, f_{3}$ and $p_{1}, p_{2}, p_{3}$ have been obtained, according to Eq. (10) the characteristic frequency $f_{\mathrm{T}}$ of the target can be resolved as follows:

$$
f_{\mathrm{T}}=\frac{f_{1} p_{2} p_{3}-2 f_{2} p_{1} p_{3}+f_{3} p_{1} p_{2}}{p_{2} p_{3}-2 p_{1} p_{3}+p_{1} p_{2}} .
$$

\subsection{Absolute velocity}

According to the principle of trigonometric function and using the measured values of $t_{1}, t_{2}$, and Eq. $(7)^{2}+$ Eq. $(8)^{2}=1$, we have

$$
\left\{\begin{array}{l}
\frac{\left(f_{1}-f_{\mathrm{T}}\right)^{2}}{f_{\mathrm{T}}^{2}} \frac{c^{2}}{v^{2}}+\frac{p_{1}^{2} D^{2}}{A_{\mathrm{T}}^{2}}=1, \\
\frac{\left(f_{2}-f_{\mathrm{T}}\right)^{2}}{f_{\mathrm{T}}^{2}} \frac{c^{2}}{v^{2}}+\frac{p_{2}^{2} D^{2}}{A_{\mathrm{T}}^{2}}=1 .
\end{array}\right.
$$

Eq. (12) can also be expressed in a matrix:

$$
\left[\begin{array}{ll}
\frac{\left(f_{1}-f_{\mathrm{T}}\right)^{2}}{f_{\mathrm{T}}^{2}} c^{2} & p_{1}^{2} \\
\frac{\left(f_{2}-f_{\mathrm{T}}\right)^{2}}{f_{\mathrm{T}}^{2}} c^{2} & p_{2}^{2}
\end{array}\right]\left[\begin{array}{c}
\frac{1}{v^{2}} \\
\frac{D^{2}}{A_{\mathrm{T}}^{2}}
\end{array}\right]=\left[\begin{array}{l}
1 \\
1
\end{array}\right] .
$$

The velocity $v$ and $D / A_{\mathrm{T}}$ can be solved as $f_{\mathrm{T}}$ has been known.

\subsection{Closest point of approach (CPA)}

When $f_{\mathrm{T}}, v$, and $D / A_{\mathrm{T}}$ have been known, $\operatorname{tg} \varphi_{i}$ can be calculated on the basis of Eq. (9). Then the closest point of approach $D$ can be derived from Eq. (6) by use of the two values $\operatorname{tg} \varphi_{1}$ and $\operatorname{tg} \varphi_{2}$. Finally, the absolute amplitude $A_{\mathrm{T}}$ at the distance which is equivalent to the unit distance from the target can be resolved.

\section{Algorithm improvement}

Only three measured data have been used in the method mentioned above. To improve the calculation accuracy in practice, the best results can be obtained by using the statistical method with more data.

\subsection{Improvement of target characteristic frequency}

For the characteristic frequency of the target, the arithmetic average of multi-point calculation results $f_{\mathrm{T} 1}, f_{\mathrm{T} 2}, \ldots, f_{\mathrm{T} n}$ that can be obtained from Eq. (11) is used as the final characteristic frequency.

$$
f_{\mathrm{T}}=\frac{1}{N} \sum_{k=1}^{n} f_{T k} .
$$

\subsection{Improvement of target velocity}

Using the multi-point data, Eq. (13) can be rewritten as 


$$
\left[\begin{array}{cc}
\frac{\left(f_{1}-f_{\mathrm{T}}\right)^{2}}{f_{\mathrm{T}}^{2}} c^{2} & p_{1}^{2} \\
\frac{\left(f_{2}-f_{\mathrm{T}}\right)^{2}}{f_{\mathrm{T}}^{2}} c^{2} & p_{2}^{2} \\
\vdots & \vdots \\
\frac{\left(f_{n}-f_{\mathrm{T}}\right)^{2}}{f_{\mathrm{T}}^{2}} c^{2} & p_{n}^{2}
\end{array}\right]\left[\begin{array}{c}
\frac{1}{v^{2}} \\
\frac{D^{2}}{A_{\mathrm{T}}^{2}}
\end{array}\right]=\left[\begin{array}{c}
1 \\
1 \\
\vdots \\
1
\end{array}\right]
$$

For

$$
\begin{gathered}
\boldsymbol{A}=\left[\begin{array}{cc}
\frac{\left(f_{1}-f_{\mathrm{T}}\right)^{2}}{f_{\mathrm{T}}^{2}} c^{2} & p_{1}^{2} \\
\frac{\left(f_{2}-f_{\mathrm{T}}\right)^{2}}{f_{\mathrm{T}}^{2}} c^{2} & p_{2}^{2} \\
\vdots & \vdots \\
\frac{\left(f_{n}-f_{\mathrm{T}}\right)^{2}}{f_{\mathrm{T}}^{2}} c^{2} & p_{n}^{2}
\end{array}\right], \\
\boldsymbol{X}=\left[\begin{array}{c}
\frac{1}{v^{2}} \\
\frac{D^{2}}{A_{\mathrm{T}}^{2}}
\end{array}\right], \text { and } \boldsymbol{B}=\left[\begin{array}{c}
1 \\
1 \\
\vdots \\
1
\end{array}\right],
\end{gathered}
$$

then in the least squares sense, there is

$$
\boldsymbol{X}=\left(\boldsymbol{A}^{\mathrm{T}} \boldsymbol{A}\right)^{-1} \boldsymbol{A}^{\mathrm{T}} \boldsymbol{B} .
$$

\section{Computer simulation}

\subsection{Error-free simulation}

First of all, the correctness of the formula will be verified by use of the computer simulation without consideration of any error.

We set the target speed of $5 \mathrm{~m} / \mathrm{s}$, characteristic line spectrum of $1000 \mathrm{~Hz}$, radiation sound source level of $134 \mathrm{~dB}$, and the equivalent voltage of $1 \mathrm{~m}$ away from the target $A_{\mathrm{T}}=501.90 \mathrm{~V}$ (which is calculated on the condition that the sensitivity of acoustic receiving transducer is $-203 \mathrm{~dB}$, and the gain of the preamplifier circuit is $120 \mathrm{~dB}$ ).

LOFAR is located in the origin of the coordinates, and the closest distance $D$ to the target is $100 \mathrm{~m}$, $1000 \mathrm{~m}$, and $10000 \mathrm{~m}$. Matlab is used as the simulation software. The sample rate of the frequency and amplitude is $1 \mathrm{~Hz}$, sound speed is $1500 \mathrm{~m} / \mathrm{s}$, and time zero is set at the moment that the target passes by the CPA point (the CPA point in Fig. 1). The information of Doppler and amplitude of the targets which contain $-300 \mathrm{~s},-200 \mathrm{~s}$, and $-100 \mathrm{~s}$ is used for the calculation.

The calculated results of the simulation model and sample data without errors are shown in Table 1.

Table 1 Calculated results without error

\begin{tabular}{ccccc}
\hline $\begin{array}{c}\text { Actual } \\
\text { closest } \\
\text { distance } \\
(\mathrm{m})\end{array}$ & $\begin{array}{c}\text { Frequency } \\
(\mathrm{Hz})\end{array}$ & $\begin{array}{c}\text { Velocity } \\
(\mathrm{m} / \mathrm{s})\end{array}$ & $\begin{array}{c}\text { Closest } \\
\text { distance } \\
(\mathrm{m})\end{array}$ & $\begin{array}{c}\text { Absolute } \\
\text { amplitude } \\
(\mathrm{V})\end{array}$ \\
\hline 100.00 & 999.99 & 5.00 & 100.00 & 501.90 \\
1000.00 & 1000.00 & 4.99 & 999.99 & 501.90 \\
10000.00 & 1000.00 & 4.99 & 9999.99 & 501.90 \\
\hline
\end{tabular}

Because this algorithm is theoretically accurate and error-free, the calculation error depends entirely on the error of the calculation process. Matlab acted as the numerical simulation software due to its high calculation accuracy. In the actual process, the error of each calculated result can reach $10^{-14}$ orders of magnitude. Table 1 does not list all the significant figures as it is limited by the table size. As in the case of $1000.00 \mathrm{~m}$, the actual calculated velocity is 4.99999999999998, and the distance is 1000.0000000000024. The correctness of this algorithm can be proved from the simulation results.

\subsection{Simulation with error}

Parameters of simulation with error are consistent with those of error-free simulation in section 5.1. The measurement error of frequency and amplitude is given below. Liu and Lei (1993) showed that in the deep sea the marine environment noise level is about $55 \mathrm{~dB}$ near the frequency of $1000 \mathrm{~Hz}$, and the equivalent noise voltage is $0.0563 \mathrm{~V}$ (the transducer sensitivity is $-203 \mathrm{~dB}$, and the gain of the preamplifier circuit is calculated as $120 \mathrm{~dB}$ ). In the simulation, the amplitude of the measured signal is given as follows:

$$
p(k)=\frac{501.902}{r(k)}+n_{\mathrm{A}},
$$

where $p(k)$ is the magnitude of the signal at time $k, r(k)$ is the distance from the target to the LOFAR at time $k$, and $n_{\mathrm{A}}$ is the Gaussian white noise with mean square error of $0.0536 \mathrm{~V}$ and with a mean of zero. 
At present, the detection of the frequency is mainly using an adaptive line spectrum enhancer, which can greatly improve the frequency measurement under the colored noise. Its measurement accuracy can reach $0.1 \mathrm{~Hz}$, which is given by

$$
f(k)=f_{\mathrm{T}}(k)+n_{\mathrm{F}},
$$

where $f(k)$ is the measured value of the frequency at time $k, f_{\mathrm{T}}(k)$ is the real value of the target's frequency at time $k$, and $n_{\mathrm{F}}$ is the Gaussian white noise with a mean square error of 0.1 and mean of zero.

Eqs. (14) and (16) are applied when calculating the target parameters. The calculated results and the relative errors of the calculated results are shown in Table 2.

As shown in Table 2, the calculated results are very accurate at close range $(1000.00 \mathrm{~m})$. The maximum relative error of distance is $2.91 \%$, which can meet the actual requirements. Considering that we are only using a single piece of passive LOFAR, such results are still satisfactory.

Table 2 Calculated results with noise

\begin{tabular}{ccccc}
\hline \multirow{2}{*}{$\begin{array}{c}\text { Actual } \\
\text { closest } \\
\text { distance } \\
(\mathrm{m})\end{array}$} & $\begin{array}{c}\text { Frequency } \\
(\mathrm{Hz})\end{array}$ & $\begin{array}{c}\text { Velocity } \\
(\mathrm{m} / \mathrm{s})\end{array}$ & $\begin{array}{c}\text { Closest dis- } \\
\text { tance }(\mathrm{m})\end{array}$ & $\begin{array}{c}\text { Absolute } \\
\text { amplitude (V) }\end{array}$ \\
\hline 100.00 & 1000.97 & 5.02 & 101.77 & 495.89 \\
& $(0.10 \%)$ & $(0.40 \%)$ & $(1.77 \%)$ & $(1.22 \%)$ \\
1000.00 & 998.96 & 5.20 & 970.82 & 483.26 \\
& $(0.10 \%)$ & $(4.00 \%)$ & $(2.91 \%)$ & $(7.12 \%)$ \\
10000.00 & 1003.32 & 6.33 & 8505.25 & 590.25 \\
& $(0.33 \%)$ & $(26.60 \%)$ & $(14.95 \%)$ & $(17.58 \%)$ \\
\hline
\end{tabular}

Data in parenthesis are relative errors

The error increases dramatically at far distance, e.g., in the case of $10000.00 \mathrm{~m}$, the calculated error of the velocity is up to $26.60 \%$. The main reason is that the variation of the target's frequency and amplitude is reduced with the increasing distance, and the variation is almost the same as that of the amplitude of the noise. Therefore, the calculated error is very large, and may even result in a singular matrix which cannot be calculated.

\section{Experiment in the air}

\subsection{Principle of the experiment}

A straight road was selected to be the experiment site, and a car was used to simulate the moving target with high-speed. The experimental equipment was divided into two parts: signal generation and acquisition. The car was used as the carrier of the signal generator which can produce a single frequency signal. The signal through a power amplifier would drive the high-power speaker to simulate the target with a single line spectrum. The signal acquisition part consisted of a high-sensitivity microphone, filter amplifier, spectrum analyzer, data logger, and oscilloscope. The microphone picked up the ambient noise in the air and the characteristic frequency of the target. After filtering and amplification, the data was finally collected and stored by the data acquisition instrument. The spectrum analyzer and oscilloscope as the monitoring devices were used to observe the noise signal in the frequency domain and time domain (Fig. 2).

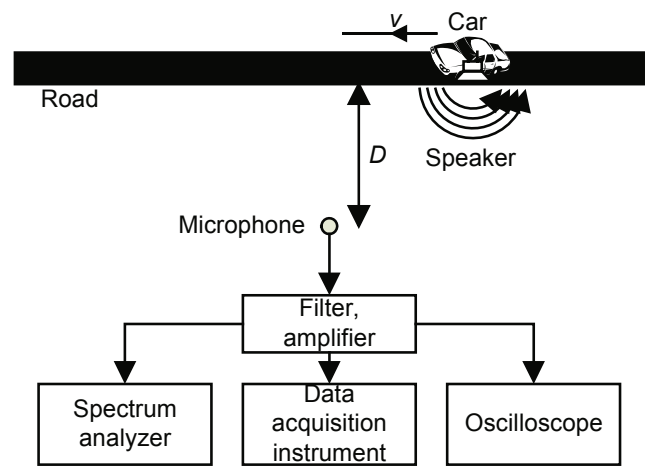

Fig. 2 Diagram of experiment plan

In the experiment (Fig. 2), the car traveling at a constant speed on the highway can simulate the targets which passed by the microphone, namely the CPA point at a uniform speed. The signal acquisition part collected the environmental noise and the target line spectrum which were stored by the data logger.

The experiment content was mainly to change the different distances of the CPA ( $D$ in Fig. 2$)$ and the car speed $v$, and then the collected sound data were used to calculate the frequency $f$ of the signal, the absolute amplitude $A$, the speed $v$ of the car, and the CPA distance $D$. The correctness of the algorithm and the effects of different factors on the algorithm will be verified in the experiment.

\subsection{Processing method}

The collected noise spectrum is a time domain signal. To estimate the frequency of each time accurately, the short-time Fourier transform method is 
used. In each fast Fourier transform (FFT), the length is to be 256 sampling points, the FFT overlap rate is $50 \%$, and the length of the FFT algorithm is 1024 . When the FFT of each time period is obtained, the frequency point with the maximum amplitude will be chosen as the measured frequency in this time period. The calculated frequency signal is to be handled by smooth filtering, namely using a 32-order finite impulse response (FIR) low-pass filter whose low-pass cut off frequency is $100 \mathrm{~Hz}$.

\subsection{Experiment results and analysis}

In the process of the experiment, the microphone sensitivity is $-45 \mathrm{~dB}$, and the microphone frequency response band is $50 \mathrm{~Hz}-18 \mathrm{kHz}$. The gain of the amplifier is $60 \mathrm{~dB}$, and the pass band frequency range of the filter is $0-20000 \mathrm{~Hz}$. The sampling rate of the data logger is $50000 \mathrm{~Hz}$. Four different CPAs are selected in the experiment, i.e., 7, 11.6, 15, and $20 \mathrm{~m}$ (due to the environmental limitation of the actual road, the maximum distance is $20 \mathrm{~m}$ ). The speed of the car is respectively selected as $20,40,60$, and $80 \mathrm{~km} / \mathrm{h}$. The frequency of the signal is $1000 \mathrm{~Hz}$. The battery is chosen to be the energy source to drive the signal whose amplitude remains constant with the root mean square (RMS) of $15.55 \mathrm{~V}_{\text {RMS }}$. The speed of
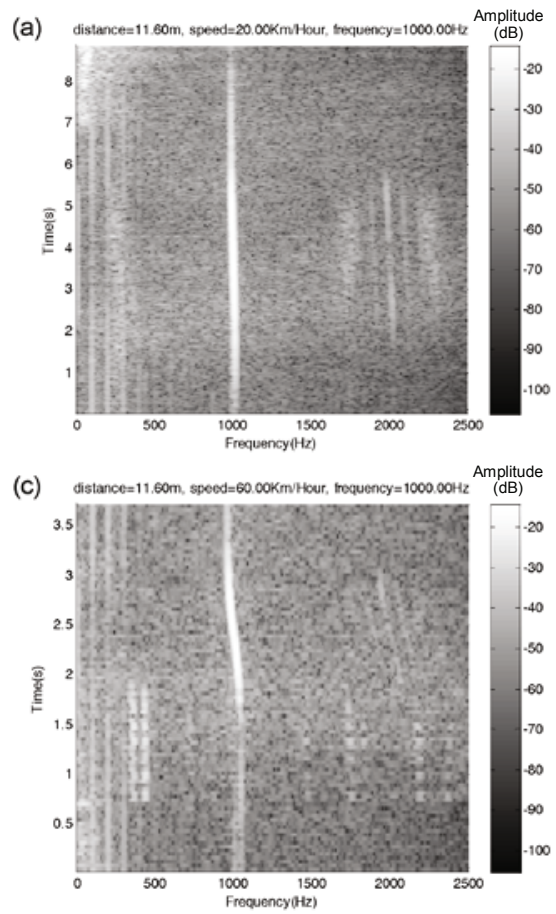

sound in the air is taken as $c=340 \mathrm{~m} / \mathrm{s}$ while processing the data.

Some typical processed results are shown in Fig. 3. Fig. 3 is the time-frequency cumulative results, in which the target passes by the CPA point whose distance is $11.60 \mathrm{~m}$, the speed is respectively set as 20 , 40,60 , and $80 \mathrm{~km} / \mathrm{h}$, and the characteristic frequency is $1000 \mathrm{~Hz}$. The higher the brightness of the figure is, the greater the amplitude of the signal means. When the vehicle approaches, the frequency of the signal is greater than the actual frequency $(1000 \mathrm{~Hz})$, and the Doppler is positive. When the car departs, the frequency of the signal is decreasing, and the Doppler is negative.

The turning points at the frequency curve indicate the time when the target passed by the CPA point. It can be seen that the change rate of the CPA point is greater when the speed of the vehicle is higher.

There are totally 13 times valid processes in the entire experiment. The frequency and the amplitude of the signal are handled on the computer. The results are shown in Table 3.

The experiment has achieved good results (Table 3). Most of the processes (except the experiment No. 6) estimate the motion parameters of the target well. The relative error of the calculated distance has
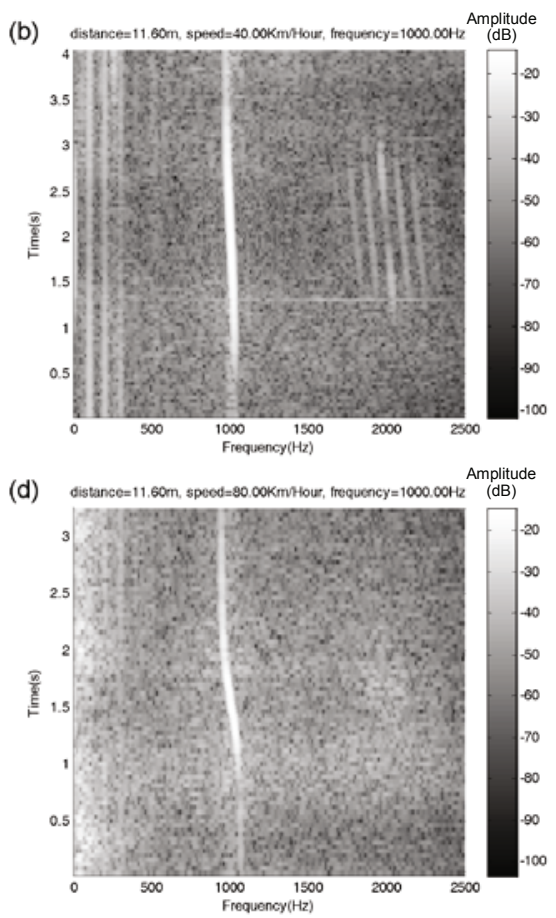

Fig. 3 Processed results (a)-(d) of time-frequency 
an average value of $3.95 \%$ and the maximum value of $9.90 \%$, while the relative error of the calculated frequency has an average value of $0.19 \%$ and the maximum value of $0.38 \%$. The relative error of the calculated velocity has an average value of $2.85 \%$ and the maximum value of $4.77 \%$, and the relative error of the calculated amplitude has an average value of $3.19 \%$ and the maximum value of $9.71 \%$.

According to the analysis of the experiment results, the closest distance has the greatest impact on the calculation error, which is consistent with the results of the computer simulation. The greater the distance is, the larger the calculation error will be. The most accurate estimated parameter is frequency, due to the frequency being stronger in antiinterference ability than other parameters. At the same time, there is no error propagation in the calculation process, when this method is directly used to measure the frequency. The estimation accuracy of velocity is higher than that of the distance and absolute amplitude, because the velocity is directly due to the Doppler frequency shift. The accuracy of the velocity will decrease, if the accuracy of the frequency is lower. The estimation accuracy of the closest distance and the absolute amplitude is the worst one. The distance calculation depends on the change of the frequency. When the distance is far, the relative velocity is reduced, and the change rate of the frequency decreases, which is equal to the reduction of the signal noise ratio (SNR). Therefore, the accuracy of the calculated results is reduced. Moreover, for the amplitude of the signal, the external interference is added directly to the amplitude information, leading to poor estimation accuracy. For example, in the experiment of No. 6, a heavy truck passed by, so some strong broadband interference was introduced in the vicinity of the low frequency band of $300-500 \mathrm{~Hz}$ at the time of $0.075 \mathrm{~s}-0.18 \mathrm{~s}$. The strong interference, shown in Fig. 3c, resulted in the reduction of the calculation accuracy of the parameter.

\section{Conclusions}

In this paper, radiated noise's amplitude information of the target was added to the parameter calculation process of LOFAR firstly. In theory, the error-free formulas were derived by using the amplitude and frequency information of the target, through any three equal interval measurements. Four important parameters, that is the speed of the target, the closest distance, characteristic frequency, and absolute amplitude, were obtained.

The method does not need to overcome the disadvantages of the previous methods. The calculation is completed without the requirement of the CPA point. It improves the calculation speed and efficiency of the LOFAR, and makes the tactics more flexible. Moreover, it is an error-free method in theory, and has important theoretical significance in the algorithm research of the LOFAR fields.

The simulation and experimental results in air show the validity of the method. Relative error of each estimated parameter is less than $10 \%$. Moreover,

Table 3 Calculated parameters in air experiment

\begin{tabular}{ccccccccc}
\hline $\begin{array}{c}\text { Serial } \\
\text { No. }\end{array}$ & $\begin{array}{c}\text { Actual } \\
\text { distance } \\
(\mathrm{m})\end{array}$ & $\begin{array}{c}\text { Actual } \\
\text { frequency } \\
(\mathrm{Hz})\end{array}$ & $\begin{array}{c}\text { Actual } \\
\text { velocity } \\
(\mathrm{km} / \mathrm{h})\end{array}$ & $\begin{array}{c}\text { Actual amplitude } \\
\text { of the signal } \\
\left(\mathrm{V}_{\mathrm{RMS}}\right)\end{array}$ & $\begin{array}{c}\text { Calculated } \\
\text { distance } \\
(\mathrm{m})\end{array}$ & $\begin{array}{c}\text { Calculated } \\
\text { frequency } \\
(\mathrm{Hz})\end{array}$ & $\begin{array}{c}\text { Calculated } \\
\text { velocity } \\
(\mathrm{km} / \mathrm{h})\end{array}$ & $\begin{array}{c}\text { Calculated } \\
\text { amplitude } \\
\left(\mathrm{V}_{\mathrm{RMS}}\right)\end{array}$ \\
\hline 1 & 7.0 & 1000 & 80 & 15.55 & 7.03 & 1002.98 & 78.04 & 15.54 \\
2 & 7.0 & 1000 & 60 & 15.55 & 6.87 & 1001.25 & 62.68 & 15.20 \\
3 & 7.0 & 1000 & 40 & 15.55 & 6.95 & 999.50 & 41.91 & 15.98 \\
4 & 11.6 & 1000 & 20 & 15.55 & 11.01 & 996.25 & 20.04 & 16.01 \\
5 & 11.6 & 1000 & 40 & 15.55 & 11.46 & 1002.05 & 38.13 & 15.67 \\
6 & 11.6 & 1000 & 60 & 15.55 & 10.24 & 997.43 & 57.43 & 14.28 \\
7 & 11.6 & 1000 & 80 & 15.55 & 11.50 & 1001.24 & 79.29 & 15.64 \\
8 & 15.0 & 1000 & 80 & 15.55 & 15.57 & 995.63 & 77.68 & 16.06 \\
9 & 15.0 & 1000 & 60 & 15.55 & 16.05 & 1001.45 & 58.36 & 14.90 \\
10 & 15.0 & 1000 & 40 & 15.55 & 15.33 & 1002.54 & 38.46 & 15.90 \\
11 & 20.0 & 1000 & 60 & 15.55 & 21.98 & 1001.20 & 58.03 & 15.99 \\
12 & 20.0 & 1000 & 40 & 15.55 & 18.74 & 999.35 & 39.79 & 16.58 \\
13 & 20.0 & 1000 & 80 & 15.55 & 18.38 & 998.59 & 77.22 & 14.04 \\
\hline
\end{tabular}


this method only uses one single piece of LOFAR, with the accurate estimation of the target distance, speed, frequency, and amplitude at close range. Hence, it has a very good application prospects for many engineering fields, such as road speed measurement, namely using a single microphone to measure the speed of a vehicle and amplitude level of the noise. Further research will focus on the case of how to improve the estimation accuracy of the target parameter over a long distance.

\section{References}

Brian, G.F., Kam, W.L., 2002. Passive ranging errors due to multipath distortion of deterministic transient signals with application to the localization of small arms fire. The Journal of the Acoustical Society of America, 111(1): 117-128. [doi:10.1121/1.1402619]

Cockrell, K.L., Schmidt, H., 2010. Robust passive range estimation using the waveguide invariant. The Journal of the Acoustical Society of America, 127(5):2780-2789. [doi:10.1121/1.3337223]

Fan, Y.Y., Tao, B.Q., Xiong, K., et al., 2002. Feature extraction of ship radiated noise by $1 \frac{1}{2}$-spectrum. Acta Acustica, 02:137-145.

Hu, Z.X., Sun, M.T., Su, W.G., 2009. Simulation analysis of LOFIX fixing accuracy for passive omnidirectional sonobuoy. Electronics Optics \& Control, 16(12):26-29.

Li, J., Sun, G.Q., Han, Q.B., 2012. Acoustics vector sensor linear array passive ranging based on waveguide invariant Proceedings of the $3 \mathrm{rd}$ International Conference on Ocean Acoustics, p.576-586.

Ling, G.M., Wang, Z.M., 2007. Sonobuoy technology and its development direction. Acoustic and Electronic Engineering, 3:1-5 (in Chinese).
Liu, B.S., Lei, J.Y., 1993. Principle of Underwater Acoustics. Harbin Engineering University Press, Harbin, China, p.34, 72, 227 (in Chinese).

Ma, J.G., 2007. Passive Localization Technology of Time Reversal. PhD Thesis, Harbin Engineering University, China (in Chinese).

Mao, W.N., 2001. An overview of passive localization for under-water acoustics. Journal of Southeast University, 31(6):1-4.

Shang, E.C., Clay, C.S., Wang, Y.Y., 1985. Passive harmonic source ranging in waveguides by using mode filter. The Journal of the Acoustical Society of America, 78(1): 172-175. [doi:10.1121/1.392554]

Sun, H., Sun, M.T., Liu, J.L., 2010. Simulation analysis on HYFIX locating accuracy of passive omni-directional sonobuoy. Electronics Optics \& Control, 17(1):85-88.

Tao, L.W., 2009. Study on Array Sonobuoy Signal Processing System and Algorithm. PhD Thesis, Northwestern Polytechnical University, Xi'an, China (in Chinese).

Tao, L.W., Wang, Y.M., 2008. New algorithm for sonobuoy Doppler-CPA. Journal of System Simulation, 20(23): 6353-6355.

Wu, Y.F., Sun, N.H., 1999. The key equipment of submarine: present situation and development of sonobuoy. Technical Acoustics, 18:95-96.

Xue, S.H., Ye, Q.H., Huang, H.N., et al., 2005. Passive-range estimation using near-field MVDR dual focused beamformers. Applied Acoustics, 24(3):177-181.

Yang, P., Yuan, B.C., Zhou, S., 2010. A line spectrum estimation method of underwater target radiated noise base on the $1 \frac{1}{2} \mathrm{D}$ spectrum. International Conference on Innovative Computing and Communication and Asia-Pacific Conference on Information Technology and Ocean Engineering, p.297-299. [doi:10.1109/CICC-ITOE.2010. 82]

Yu, T., 2011. Doppler direct location for underwater target. Journal of China Academy of Electronics and Information Technology, 6(3):328-330 (in Chinese).

\section{中文概要：}

\section{本文题目 : 低频分析和记录场合基于幅度和频率联合测量的目标定位方法}

Target localization based on joint measurement of amplitude and frequency in a LOFAR field

研究目的 : 针对低频分析和记录 (LOFAR) 使用场合, 利用目标的幅度和频率信息来解算目标运动要素。

创新要点 : 利用目标幅度和频率信息, 推出无误差的目标特征频率、绝对速度、最接近点距离、辐射幅 度等四个参量理论公式, 并得到解算目标运动要素的优化方法。

研究方法 : 1. 用计算机进行无误差的理论验证仿真, 并在有噪声的影响下, 验证算法的正确性; 2. 在空 气中，使用汽车模拟目标的运动，用麦克风采集汽车运动的多普勒信号 (见图 2), 验证算法 的工程有效性和正确性。

重要结论：1. 使用三次等间隔采样的目标幅度和频率信息, 即可无误差地解算出目标特征频率、绝对速 度、最接近点距离、辐射幅度等四个参量; 2. 使用优化后的算法, 经实际空气实验验证, 使 用单个 LOFAR 传感器即可解算出目标的四个参量, 且四个参量的估计相对误差小于 $10 \%$ 。 